\title{
BMJ Open Lenient rate control versus strict rate control for atrial fibrillation: a protocol for the Danish Atrial Fibrillation (DanAF) randomised clinical trial
}

\author{
Joshua Buron Feinberg (D) ,, ${ }^{1,2}$ Michael Hecht Olsen, ${ }^{1,2}$ Axel Brandes, ${ }^{3}$ \\ Ilan Raymond, ${ }^{1}$ Walter Bjørn Nielsen, ${ }^{1}$ Emil Eik Nielsen, ${ }^{1,4}$ \\ Frank Stensgaard-Hansen, ${ }^{1}$ Ulrik Dixen, ${ }^{5}$ Ole Dyg Pedersen, ${ }^{6}$ \\ Uffe Jakob Ortved Gang, ${ }^{6}$ Christian Gluud, ${ }^{2,4}$ Janus Christian Jakobsen ${ }^{2,4}$
}

To cite: Feinberg JB, Olsen MH, Brandes A, et al. Lenient rate control versus strict rate control for atrial fibrillation: a protocol for the Danish Atrial Fibrillation (DanAF) randomised clinical trial. BMJ Open 2021;11:e044744. doi:10.1136/ bmjopen-2020-044744

- Prepublication history and additional material for this paper is available online. To view these files, please visit the journal online (http://dx.doi.org/10. 1136/bmjopen-2020-044744)

Received 11 September 2020 Revised 18 February 2021 Accepted 28 February 2021
Check for updates

(C) Author(s) (or their employer(s)) 2021. Re-use permitted under CC BY-NC. No commercial re-use. See rights and permissions. Published by BMJ.

For numbered affiliations see end of article.

Correspondence to Dr Joshua Buron Feinberg; wtv945@alumni.ku.dk

\section{ABSTRACT}

Introduction Atrial fibrillation is the most common heart arrhythmia with a prevalence of approximately $2 \%$ in the western world. Atrial fibrillation is associated with an increased risk of death and morbidity. In many patients, a rate control strategy is recommended. The optimal heart rate target is disputed despite the results of the the RAte Control Efficacy in permanent atrial fibrillation: a comparison between lenient vs strict rate control II (RACE II) trial.

Our primary objective will be to investigate the effect of lenient rate control strategy ( $<110$ beats per minute (bpm) at rest) compared with strict rate control strategy (<80 bpm at rest) on quality of life in patients with persistent or permanent atrial fibrillation.

Methods and analysis We plan a two-group, superiority randomised clinical trial. 350 outpatients with persistent or permanent atrial fibrillation will be recruited from four hospitals, across three regions in Denmark. Participants will be randomised 1:1 to a lenient medical rate control strategy ( $<110 \mathrm{bpm}$ at rest) or a strict medical rate control strategy $(<80 \mathrm{bpm}$ at rest). The recruitment phase is planned to be 2 years with 3 years of follow-up. Recruitment is expected to start in January 2021. The primary outcome will be quality of life using the Short Form-36 (SF-36) questionnaire (physical component score). Secondary outcomes will be days alive outside hospital, symptom control using the Atrial Fibrillation Effect on Quality of Life, quality of life using the SF-36 questionnaire (mental component score) and serious adverse events. The primary assessment time point for all outcomes will be 1 year after randomisation.

Ethics and dissemination Ethics approval was obtained through the ethics committee in Region Zealand. The design and findings will be published in peer-reviewed journals as well as be made available on ClinicalTrials.gov. Trial registration number NCT04542785.

\section{INTRODUCTION}

Atrial fibrillation is the most common arrhythmia of the heart with a prevalence of approximately $2 \%$ in the western

\section{Strengths and limitations of this study}

- First trial assessing lenient versus strict rate control in patients who upon inclusion are considered as having persistent atrial fibrillation. Hence, this trial is expected to provide data on patients who upon inclusion have a relatively short duration of atrial fibrillation.

- First superiority trial with quality of life as primary outcome in patients with both permanent atrial fibrillation and persistent atrial fibrillation on inclusion.

- Pragmatic trial with multiple sites ensuring high external validity.

- Treatment providers are not blinded in a trial that is otherwise expected to have low risk of bias regarding blinding of other domains.

- Trial will not have enough power to assess 'hard outcomes' such as mortality and serious adverse events.

world. ${ }^{12}$ Atrial fibrillation is associated with an increased risk of death and a number of morbidities. $^{3-9}$ The risks of both cerebral stroke and heart failure are increased nearly fivefold in patients with atrial fibrillation, and about $20 \%$ of all strokes may be due to atrial fibrillation. ${ }^{3-8}$ Atrial fibrillation also has a significant impact on healthcare costs and accounts for approximately $1 \%$ of the National Health Service budget in the UK and approximately $\$ 26$ dollars of annual expenses in the USA. ${ }^{1011}$

Two different overall intervention strategies may be used for atrial fibrillation: a rhythm control strategy or a rate control strategy. ${ }^{12-14}$

We have previously shown in a systematic review with meta-analysis and trial sequential analysis that rhythm control strategies compared with rate control strategies seem to significantly increase the risk of serious adverse events in 
patients with atrial fibrillation. ${ }^{13}{ }^{14}$ Based on current evidence as well as guidelines, it seems that most patients with atrial fibrillation should be treated with a rate control strategy unless there are specific reasons justifying a rhythm control strategy. ${ }^{1314}$

The resting heart rate target for rate control has recently changed from below 80 beats per minute (bpm) to below 100-110 bpm at rest depending on the guideline. ${ }^{12} 1415$ This change was a result of the the RAte Control Efficacy in permanent atrial fibrillation: a comparison between lenient vs strict rate control II (RACE II) trial, which randomised 614 participants to a lenient rate control strategy $(<110 \mathrm{bpm}$ at rest) versus a strict rate control strategy $\left(<80 \mathrm{bpm}\right.$ at rest) ${ }^{16}$ The participants were outpatients with permanent atrial fibrillation. The RACE II trial showed that the lenient rate control strategy was non-inferior compared with the strict rate control strategy on the risk of a composite outcome of mortality, stroke, cardiac arrest, arrhythmic events, systematic emboli or major bleeding. Furthermore, the HR of 0.84 (90\% CI 0.58 to 1.21) suggested that the lenient rate control group might decrease the risk of the composite outcome. The RACE II trial also showed no difference of the two strategies on quality life, but this analysis has questionable validity. ${ }^{17}$

A theoretical concern when using a lenient control strategy is that patients may develop heart failure if the heart rate is too fast. ${ }^{18-20}$ The RACE II trial found that the lenient strategy was also non-inferior for heart failure patients but the majority of the participants had preserved EF at baseline. ${ }^{21}$

We searched the Cochrane Central Register of Controlled Trials, MEDLINE and ClinicalTrials.gov on 26 September 2019. Our literature search identified only the RACE II trial assessing the effect of lenient rate control versus strict rate control in atrial fibrillation. We found no systematic reviews or meta-analyses on the topic.

\section{Trial rationale}

Currently, lenient rate control is the guideline recommended initial rate control strategy. ${ }^{14}$ However, this recommendation is primarily based on the RACE II trial, which had two major limitations. First, the validity of the RACE II trial results when assessing symptoms and quality of life were questionable mainly because of substantial problems with missing data. Regarding quality of life and symptom severity, only $437 / 614(71 \%)$ participants had data available at maximum follow-up. ${ }^{17}$ Furthermore, the authors did not use multiple imputation or other valid methods to handle the missing data. ${ }^{22}$ Second, the RACE II trial only showed a lenient rate control strategy was non-inferior but could not answer if a lenient rate control strategy is superior to a strict rate control strategy. The RACE II trial was not adequately powered to confirm or reject minimal important differences between the two strategies. Conducting a superiority randomised clinical trial and afterwards performing a systematic review with meta-analysis will give us the possibility of confirming or rejecting that there is a difference in effect between the two strategies, at least on quality of life.

\section{Health-related quality of life as an outcome}

There are many definitions of health-related quality of life. ${ }^{2324}$ In general, quality of life questionnaires can be designed in two ways. ${ }^{23}$ Generic questionnaires assess multiple domains applicable to a variety of health domains. ${ }^{23}$ They more readily permit comparison across different disease and seem to have unquestionable patient relevance. ${ }^{23}{ }^{25}$ Generic quality of life scales are often criticised for being less sensitive to change than disease-specific quality of life scales, but when outcome results show no difference, it is most often unknown whether the lack of difference is caused by non-sensitive outcome scales or if the results demonstrate that there is no 'true' difference between the compared interventions when assessing 'generic' quality of life. ${ }^{23} 25$ The opposite holds true for disease-specific questions, which in general are thought to be more responsive to change in the clinical condition than generic disease questionnaires but may be less patient relevant. The disease-specific questionnaires tend to focus more narrowly on the disease. Any increase in quality of life as a result of a treatment for a specific disease may be off set by unforeseen negative consequences of the treatment that the questionnaire by design will not capture.

We will therefore supplement the general assessment using Short Form-36 (SF-36) with a disease-specific questionnaire. Currently, there seems to be no optimal questionnaire. ${ }^{25} 26$ The Atrial Fibrillation Effect on Quality of Life (AFEQT) is a validated, disease-specific questionnaire, which aims to capture the objective and subjective burden of disease. ${ }^{27}$ It contains 20 items that aim to assess four domains: symptoms, activities, treatment concern and treatment satisfaction. It also includes a summary score that summarises the first three domains. It assesses the burden of the atrial fibrillation symptoms. $^{2728}$

When assessing quality of life, it is important to focus on a minimally important difference, which typically can be done using an anchor-based method or a distribution-based method, or a mix of the two. ${ }^{29} 30$ To interpret the clinical significance of future trial results, we will carefully define minimal important differences for all primary and secondary outcomes (see 'Statistical plan and data analyses'). ${ }^{31}$

\section{Objectives}

Our primary objective will be to investigate the effect of a lenient rate control strategy $(<110 \mathrm{bpm}$ at rest) compared with a strict rate control strategy ( $<80 \mathrm{bpm}$ at rest) on quality of life in patients with persistent or permanent atrial fibrillation.

\section{METHODS AND ANALYSIS \\ Trial design}

The design of the Danish Atrial Fibrillation (DanAF) trial will be a randomised, two-group, superiority trial of lenient rate control versus strict rate control in patients with persistent or permanent atrial fibrillation at inclusion who accept rate control as the main strategy. Treatment providers responsible for the rate control treatment will not be blinded. Any other treatment providers (i.e. those 
managing co-morbidities) will be attempted blinded as well as participants.

Three hundred and fifty outpatients will be recruited from four university hospitals in Denmark: Holbaek University Hospital, Hvidovre University Hospital, Region Zealand University Hospital - Roskilde and Odense University Hospital.

The present protocol follows the recommendation in the Standard Protocol Items: Recommendations for Interventional Trials guideline including all items from the WHO Trial Registration Data Set (online supplemental files 1 and 2).

\section{Trial conduct}

This trial will be conducted according to good clinical research practice and the latest Declaration of Helsinki. $^{32} 33$

\section{Randomisation}

Participants will be randomised 1:1 to a lenient or a strict medical rate control strategy. The trial will use centralised randomisation at OPEN. Prior to the trial, a computer will generate randomisation sequences with varying block sizes between 6 and 10 that are unknown to the investigators. An internet-based randomisation system will be set up conducting randomisation stratified according to site, type of atrial fibrillation at inclusion (persistent vs permanent) and left ventricular ejection fraction (LVEF) (ejection fraction (EF) $\geq 40 \%$ and $\mathrm{EF}<40 \%$ ). The randomising investigator will get access to the internet site through a personal password. The randomising investigator will not be an outcome assessor.

\section{Blinding}

The investigator prescribing the rate control medication (treatment provider) will not be blinded, as the treatment requires knowledge of the group the participant is randomised to. All other treatment providers, outcome assessors, data managers, statisticians and participants will be sought blinded (the participants will neither be informed of their rate control target nor their allocated intervention group). Blinded data will be sent to OPEN for blinded data management. Statistical analyses will be performed with the two intervention groups coded as 'A' and 'B' by two independent blinded statisticians. Two blinded conclusions will be drawn by the steering group: one assuming ' $\mathrm{A}$ ' is the experimental group and ' $\mathrm{B}$ ' is the control group-and one assuming the opposite. Based on these two blinded conclusions, two abstracts will be written (will be published as a supplement to the main publication). When the blinding is broken, the 'correct' abstract will be chosen, and the conclusions in this abstract will not be revised.

As all medical procedures are available to any treatment provider, we cannot foresee any reason for unblinding participants. If, however, any medical personnel deem it necessary to unblind a participant, the participant will be unblinded.

\section{Selection of participants}

\section{Inclusion criteria}

1. Participants withatrial fibrillation (ECG confirmed and diagnosed by the treatment provider) who at inclusion have either persistent (defined as atrial fibrillation for more than 7 days) or permanent atrial fibrillation (only rate control is considered going forward).

2. Rate control must be accepted as being the primary management strategy going forward. Consideration towards whether rhythm control is more appropriate must be considered, especially given the results of the Early treatment of Atrial fibrillation for Stroke prevention Trial (EAST).$^{34}$

3. Informed consent.

4. Adult (18 years or older).

\section{Exclusion criteria}

1. No informed consent.

2. Initial heart rate under $80 \mathrm{bpm}$ at rest (assessed via ECG before randomisation).

3. Less than 3 weeks of anticoagulation with new oral anticoagulants or 4 weeks with efficient warfarin.

4. Participants dependent on a high ventricular rate to maintain a sufficient cardiac output. This will be based on an individual assessment of the possible participant. Such participants could be participants with heart failure, participants with a haemodynamically significant valve dysfunction or severely dehydrated participants. Other factors such as echocardiographic assessments, stability of the disease and similar will be factored in when judging if a participant is dependent on a high ventricular rate. Such a decision will be made before randomisation by the treatment provider.

5. Participants who are haemodynamically unstable and therefore require immediate electrical cardioversion.

\section{Participant withdrawal}

Participants can withdraw his or her consent at any time point for any reason but will be invited to still participate in the follow-up assessments.

\section{Interventions}

\section{Lenient rate control}

The heart rate will be assessed on a 12-lead resting ECG measured over $1 \mathrm{~min}$ after $5 \mathrm{~min}$ of rest. The treatment provider will target the highest tolerable resting heart rate $<110 \mathrm{bpm}$. Treatment providers are encouraged not to attempt to lower the heart rate if already below 110 unless symptoms or other reasons necessitates this. If the heart rate is below 90, the treatment provider is encouraged to reduce rate limiting treatment. If the patient remains symptomatic due to atrial fibrillation after achieving this definition of heart rate control, Holter monitoring or exercise tests may be deemed necessary by the treatment provider.

These evaluations may be followed by adjustment of rate control drugs, rhythm control (electrical cardioversion, arrhythmia surgery and rhythm control medications) 
or atrioventricular node ablation. In case of the need for rhythm control or atrioventricular node ablation, the allocated heart rate target is no longer relevant in management.

\section{Strict rate control}

Strict rate control achieved by using rate control medication (see further) will be defined as a mean resting heart rate $<80 \mathrm{bpm}$ with a general recommendation of targeting $70 \mathrm{bpm}$ on a 12-lead resting ECG measured over $1 \mathrm{~min}$ after 5 min of rest. Exercise test to determine activity heart rates or Holter monitoring will only be performed if the treatment provider believes this is indicated. These evaluations may also be followed by adjustment of rate control medications, electrical cardioversion, arrhythmia surgery or atrioventricular node ablation (treatment provider's choice).

\section{Rate control medications}

Treatment will be provided according to current guidelines, and as such, the algorithm for treatment will be differentiated based on the status of left ventricular ejection fraction. ${ }^{14}$ For participants with reduced LVEF, betablockers (metoprolol and bisoprolol) will be the primary therapy. Secondary therapies may include digoxin or amiodarone. For participants with preserved LVEF, the primary therapy will be beta-blockers (metoprolol and bisoprolol) or non-dihydropyridine calcium-channel blockers (verapamil) with secondary therapy consisting of digoxin or amiodarone.

We briefly summarise the pharmacological treatment in the DanAF trial (table 1).

\section{Concomitant medication}

Besides rate control, the treatment provider will be free to prescribe any other standard medical cointervention such as the need for anticoagulation (based on the $\mathrm{CHA}_{2} \mathrm{DS}_{2}-$ VASc score and comorbidity, ${ }^{14}$ hypertension management, heart failure management or lipid lowering drugs as long as the prescriptions adhere to guidelines. ${ }^{14}$ This also includes recommendations regarding modifiable risk factors that may have adverse effects on atrial fibrillation management (excess alcohol, smoking and sleep apnoea). ${ }^{145} \mathrm{~A}$ brief description of what is considered standard management of comorbidities and risk factors are given in online supplemental file 3 . All other interventions are allowed if they are administered evenly in all intervention arms.

\section{Table 1 Suggested daily doses for rate control agents}

Metoprolol 50-200mg

Bisoprolol 2.5-10 mg

Digoxin $\quad 62.5-250 \mu g$ maintenance dose according to weight, age and renal function; loading is usually required for 3-7 days

Verapamil 120-240 mg - no loading dose required

\section{Follow-up and outcome events}

All participants will attend a minimum of two follow-up visits within 2 months after randomisation. Further visits are possible with 2-week intervals until adequate titration of rate control therapy is as required or for other reasons such as participants having inadequate symptom control, management of comorbidities and so on. Treatment providers may plan a visit sooner or later if clinically indicated. To assess if the ECG guided heart rate target is representative of the heart rate under normal conditions, we will perform 24-hour Holter monitoring at the end of the titration phase and after 1 year of follow-up for documentation purposes.

After the initial adequate titration of rate control, participants are to follow the normal referral system in the Danish healthcare system. A hotline will be established where treatment providers may call and ask for the participant's rate control target. If treatment providers themselves do not contact the trial treatment provider, participants are encouraged to contact the trial treatment provider. If possible, a treatment provider involved in the trial will be the managing treatment provider of the referral, if the referral is to a participating department.

\section{Primary outcome}

- Quality of life using the SF-36 questionnaire (physical component score), continuous outcome. ${ }^{36}$

\section{Secondary outcomes}

- Days alive outside hospital, count outcome.

- Symptoms due to atrial fibrillation using the AFEQT, continuous outcome. ${ }^{27}$

- Quality of life using the SF-36 questionnaire (mental component score), continuous outcome. ${ }^{36}$

- Serious adverse events, dichotomous outcome. We will define a serious adverse event as any untoward medical occurrence that resulted in death, was lifethreatening, required hospitalisation or prolongation of existing hospitalisation and resulted in persistent or significant disability or jeopardised the patient. ${ }^{33}$

\section{Exploratory outcomes}

- All-cause mortality, dichotomous outcome.

- Composite of all-cause mortality, stroke, myocardial infarction and cardiac arrest, dichotomous outcome.

- Cardiac mortality, dichotomous outcome.

- Stroke, dichotomous outcome.

- Hospitalisation for worsening of heart failure, dichotomous outcome.

- Number of hospital admissions, count outcome.

- Six-minute walking distance, continuous outcome.

- Healthcare costs.

- Various biomarkers (N-terminal pro-brain natriuretic peptide (nt-proBNP), high-sensitivity $\mathrm{C}$ reactive protein (hsCRP), high-sensitivity troponin I (hsTnI), growth differentiation factor-15 (GDF-15), interleukin 6 (IL6), cystatin-C, YKL40, soluble urokinase plasminogen activator receptor (suPAR) and fibulin-1). 
- Switch to rhythm control strategy (such as rhythm control medication, DC-conversion, pulmonary vein isolation or arrhythmia surgery), dichotomous outcome.

- Implantation of a pacemaker or cardioverter-defibrillator with or without $\mathrm{AV}$ node ablation, dichotomous outcome.

\section{Echocardiographic outcomes}

- Size of left atrium (Left atrial volume index)).

- Size of left ventricle.

- Cardiac index (cardiac output/body surface area).

- Left ventricular ejection fraction.

- Tricuspid annular plane systolic excursion (TAPSE). ${ }^{37}$

- Midwall fractional shortening.

- Global longitudinal strain.

- Circumferential end-systolic stress.

- Diastolic dysfunction estimated by the relationship between left ventricular filling and the interval between two successive R waves on ECG (R-R interval) for the individual patient.

- Pulmonary pressure.

All secondary, exploratory and echocardiographic outcomes will only be hypothesis generating.

\section{Adverse events}

Participants will be asked during visits to the clinic if they had experienced any undesirable medical events.

Suspected unexpected serious adverse reactions (SUSAR) will be reported to the ethics committee within 7 days of investigators being aware of the event. Once a year, a report of all serious adverse events and serious adverse reaction will be submitted to the ethics committee.

\section{Assessment time point}

The primary assessment time point for all outcomes will be 1 year after randomisation.

\section{Procedures for screening}

Potential participants according to inclusion and exclusion criteria at Holbaek University Hospital, Hvidovre University Hospital, Region Zealand University Hospital - Roskilde and Odense University Hospital will receive an invitation to participate in the trial on a routine visit in the clinic or hospitalisation for atrial fibrillation. Possible participants will be identified by trial staff employed at the site.

\section{Procedures for informed consent}

Participants will receive printed material containing details of each study visit, the design and rational of the trial, participant rights (such as the right to withdraw), possible adverse reactions of medication and more. The printed material will be given either immediately after being identified as a possible candidate or during a private, information session where verbal information is given and the participants can ask any questions they may have. The information session will take place in an undisturbed environment. The information will be given by the project coordinator on site or medical personnel with equivalent prerequisites for conveying the project. Potential participants will be informed that they can bring a third party if they wish so. The participants will be given up to 3 weeks to consider participation depending on when they choose to schedule the information session. There will be a minimum of 48 hours from the information session to the obtaining of informed consent.

\section{Data collection}

Data will be attempted to be collected from all participants regardless of protocol adherence. Study plan and data will be as shown in table 2 .

Echocardiography will be performed according to current international guidelines. ${ }^{38}$ A detailed plan for the echocardiographic examination and recordings has been developed. The echocardiograms will be sent to a core echocardiographic reading centre at Holbaek Hospital to be assessed by one of two assessors that will be blinded.

\section{Biobank}

We will collect blood samples for a research biobank and measure: Nt-proBNP, hsCRP, hsTnI, GDF-15, IL6, Cystatin-C, YKL40, suPAR and fibulin-1. In addition to the above blood samples, we will collect the following three types of blood samples: $5 \mathrm{~mL}$ serum, $5 \mathrm{~mL}$ plasma and $5 \mathrm{~mL}$ citrat plasma to be stored for future research. Participants will be given separate information on this blood collection as well as be required to give a separate informed consent (online supplemental file 4).

\section{Data management}

All data will be sent encrypted to OPEN for management. All data on paper will be securely stored, and a copy will be sent to a computerised database.

The computerised database will be continuously checked for missing values and errors at 1-month intervals. Before a trial site begins recruitment, an internal monitoring of the following procedures will be checked: validation of inclusion and exclusion criteria, informed consent procedure, randomisation procedure and data entry into REDcap.

\section{Statistical plan and data analyses}

Sample size: quality of life using the SF-36 questionnaire (physical component score)

Using a minimal important difference of 3 points on the physical component score, an SD of 10 , power of $80 \%$ and a significance level of $5 \%$ and a total of 350 participants will be needed. ${ }^{17940}$ Based on this sample size, we have estimated the power of all remaining outcomes (see online supplemental file 5).

\section{Recruitment plans}

We will involve key medical personnel at the different departments as well as hold sessions at the different departments informing of the trial. 


\begin{tabular}{|c|c|c|c|c|c|}
\hline Schedule & Visit 0 baseline & Visit 1 & Visit 2 & Visit 3 & Visits 4,5 and 6 \\
\hline Investigations & 0 months & 1 month \pm 2 week & 2 months \pm 2 weeks & 6 months \pm 2 weeks & $\begin{array}{l}12 \text { months } / 24 \\
\text { months } / 36 \text { months } \\
\pm 2 \text { weeks }\end{array}$ \\
\hline Medical history & $x$ & $x$ & & & $x$ \\
\hline $\begin{array}{l}\text { Clinical events (hospital, tests } \\
\text { and so on) }\end{array}$ & & $x$ & $x$ & & $x$ \\
\hline EHRA SC & $x$ & $x$ & $x$ & & $x$ \\
\hline SF-36 and AFEQT & $x$ & & & & $x$ \\
\hline Physical examination & $x$ & & & & $x$ \\
\hline Vital signs (BP and HR) & $x$ & $x$ & $x$ & & $x$ \\
\hline Randomisation & $x$ & & & & \\
\hline $\begin{array}{l}\text { Clinical laboratory tests (as } \\
\text { indicated) }\end{array}$ & $x$ & $x$ & $x$ & & $x$ \\
\hline Study laboratory tests & $x$ & & & $x$ & $x$ \\
\hline 12-lead ECG & $x$ & $x$ & $x$ & & $x$ \\
\hline $\begin{array}{l}\text { Holter monitoring. } 0=\text { as } \\
\text { clinically indicated }\end{array}$ & $(x)$ & $(x)$ & $x$ & & $x$ \\
\hline Echocardiography & $x$ & & & & $x$ \\
\hline Six-minute walking test & $x$ & & & & $x$ \\
\hline
\end{tabular}

AFEQT, the atrial fibrillation effect on quality of life; BP, blood pressure; $\mathrm{CHA}_{2} \mathrm{DS}$, VASc score, Score for determining the risk of stroke. Points are given for congestive heart failure (1), hypertension (1), age 75 or above (2), diabetes (1) previous stroke (2), vascular disease (1), age 65-75 (1) and female sex (1); EHRA SC, European heart rhythm association symptom classification; HR, heart rate; SF-36, Short Form-36.

\section{Statistical analyses}

A detailed statistical analysis plan will be published around 1 month after the trial has been launched. In short, our primary conclusions will be based on the results of our single primary outcome. Hence, we will consider a $p$ value of 0.05 as our threshold for statistical significance. ${ }^{31}$ The results of secondary outcomes, exploratory outcomes, subgroup analyses and possible per protocol analyses will be hypothesis generating only. We will assess whether the thresholds for statistical and clinical significance are crossed according to the five-step procedure proposed by Jakobsen et al. ${ }^{31}$ The analyses of the outcomes will be based on the 'intention to treat' principle, that is, all randomised participants will be included in the analysis regardless of how much treatment they have received. In case of more than $5 \%$ not receiving the allocated heart rate target, we will secondarily analyse all outcomes according to the actual heart rate achieved (per protocol analysis) defined as the average heart rate on ECG after 5 min of rest. Participants who receive a rhythm control strategy (assessed by the treating physician) at our primary assessment time point will be excluded from this analysis. If outcomes are not present due to retraction of informed consent or dropout, the pattern of the missing data will be investigated. Missing data will be handled according to the recommendations proposed by Jakobsen et $a l^{22}$ In short, we will conduct a worst-best and best-worst case scenario, testing the potential impact of missing data. ${ }^{22}$ If the pattern of missing data allows it, we will also conduct multiple imputations. ${ }^{22}$

\section{Analysis methods}

Continuous outcomes will be presented as means and SD with 95\% CIs. Count outcomes will be presented as medians and IQRs. We will analyse continuous outcomes using mixed effects linear regression with 'site' as a random intercept using an exchangeable covariance matrix and type of atrial fibrillation at inclusion (persistent vs permanent) and LVEF (EF $\geq 40 \%$ and EF $<40 \%)$ as a fixed effect. ${ }^{41}$ We will analyse count data using the van Elteren's test stratifying for 'site'. ${ }^{42}$ Dichotomous outcomes will be presented as proportions of participants in each group with the event, as well as risk ratios with $95 \%$ CIs. Dichotomous outcomes will be analysed using mixed effects generalised linear models using a log link function with 'site' as a random intercept using an exchangeable covariance matrix, and type of atrial fibrillation will be included as a fixed effect. ${ }^{42}$ All outcomes will be analysed according to final value. 


\section{Subgroup analyses}

All subgroup analyses will be regarded as hypothesis generating only, and we will not base any conclusions on these. We will in the planned statistical analysis plan (see 'Statistical analysis') in detail describe each planned subgroup analysis.

In short, we will in each publication compare:

- Patients with heart failure compared with patients without heart failure (including subtypes).

- Men compared with women.

- Different durations of atrial fibrillation at randomisation.

- Less than 1 year.

- 1-2 years.

- More than 2 years.

- Patients with age above compared with below 75 years.

- Patients according to the European Heart Rhythm Association symptoms score.

\section{Data monitoring}

A data safety monitoring committee (DSMC) independent from the sponsor and the investigators will be created. The DSMC will be free of conflicts of interest. The DSMC will be responsible for conducting an interim analysis after $50 \%$ of participants have been included and monitor if the trial still holds scientific merit. The DSMC will decide when/if a new interim analysis should be performed. The DSMC will make recommendations to the steering committee whether the trial should stop or continue (further details in online supplemental file 6).

\section{Auditing}

The trial can be audited by the regional ethics committee, which is independent from the investigators and sponsor.

\section{Patient and public involvement}

Patients were invited to a workshop after the initial draft was accepted by all participating departments. They were asked to give inputs to the chosen outcomes, the written material, the relevance of the objective of the trial and any other aspects they found relevant.

Patients are anticipated to work as ambassadors after the trial results are available. We will therefore perform a second workshop to involve patients in the best strategy for dissemination.

\section{Ethics and dissemination}

The management of patients is in accordance with standard care, and as such, patients are at no greater risk compared with receiving standard care outside the trial. It is therefore ethical for patients to be part of the trial. The potential benefit for future patients is that we may uncover a superior heart target to be the goal of future management of patients with atrial fibrillation.

The trial protocol has been approved by the regional ethics committee, which is a branch of the Danish ethics committee, the regulatory body approving research in Denmark. As such, the committees are independent from the trial. The committee reviewed the full protocol, the written material for the participants, the consent form and the administered questionnaires before giving approval. The ethics committee has the option of conducting an audit of the trial if it wishes to do so. The committee must be provided with a notification of any serious adverse events including Suspected unexpected serious adverse reactions within a week as well as a yearly report of serious adverse events. Any changes to the approved protocol will be submitted and approved before continuing the trial.

Site investigators or personnel with equivalent skills will obtain informed consent from possible participants (online supplemental file 7). Additional consent will be obtained in order to store blood samples for future research.

Before enrolment of participants, screening will be done by personnel employed at the study site using the local electronic journal system. Any information collected on potential and enrolled participants will be entered directly into REDcap, using a secure connection.

The project and its data have been registered at the Region Zealand, who is the data controller. Study investigators will have access to the full data set. OPEN, who is in charge of storing the data, will also have access to the full data set. Ethics review will also have access to data on request.

Participants, who suffer harm during the trial, are insured by the the Danish Patient Compensation Association.

Trial results will be sought published in a peer-reviewed journal. We will also communicate results directly to relevant patient advocacy groups, relevant medical associations and attempted presented at relevant congresses. Aggregate data analysis will be published in a clinical trial register no later than 3 years after trial results have been collected. Data sharing will be made available on request after approval from ethics committee.

Authorship will be granted according to the recommendations from the International Committee of Medical Journal Editors. ${ }^{43}$

\section{DISCUSSION}

Our trial has several strengths. It is a pragmatic trial assessing the benefits and harms of a lenient versus a strict rate control strategy on quality of life in patients with persistent or permanent atrial fibrillation. The number of inclusion and exclusion criteria is low, and hence, the external validity will be high. Participants will be recruited from more than one site, which will further increase the external validity. We have performed a sample size estimation based on previous evidence with realistic intervention effects, we will adjust the thresholds for statistical significance if the sample size is not reached, and we have chosen only one outcome we will base conclusion on. The remaining outcomes will be considered hypothesis generating only thereby taking into account problems with multiplicity. Furthermore, we have taken measures to reduce the risks of bias from the 
allocation sequence generation, allocation concealment, blinding of outcome assessors and participants, selective outcome reporting, for-profit bias and missing outcome data. Hence, our trial will be conducted with a low risk of random errors ('play of chance') and with as low risk of systematic errors ('bias') as the trial design allows (see further) ${ }^{31}{ }^{44}$ In Denmark, a complete follow-up of all participants for death and hospitalisations is secured by an unique number given to all born in Denmark, Central Person Register.

Our trial also has limitations. The treatment providers responsible for the rate control intervention will not be blinded, which may bias our results. We will use 12-lead ECG to guide rate control therapy. Holter monitoring and measurement of the heart rate during exercise will only be used at the discretion of the investigator if deemed necessary. As such, there may be fluctuations in the heart rate we do not detect. Another limitation is that we do not have sufficient power to assess 'hard outcomes' such as mortality and serious adverse events. This will be explored in a future meta-analysis with individual patient data from the RACE II trial and other trials. The consequence may ultimately be that a superiority trial in terms of 'hard outcomes' is needed. Our results will only be generalisable to a population where rate control is considered appropriate as the main strategy going forward. The results of the EAST trial is expected to delay the initiation of rate control for many patients, and hence, our results will need to be interpreted in light of this. Yet another limitation is that participants presumably will receive different medications and procedures in the compared groups. If we show a difference (or lack of a difference) between the groups, it will be difficult to interpret what part of the treatment algorithm for reaching a certain rate target caused this difference.

We expect the results of this trial will play a part of future recommendations for rate control treatment in patients with both persistent and permanent atrial fibrillation.

\section{Protocol version and amendments}

This abbreviated version of the full protocol is based on V.2.0 of the protocol (January 2020). Any changes to the original protocol will be submitted to the regional ethics committee. After approval, changes will be conveyed to all investigators, participants and trial registries.

The findings will be published in a peer-reviewed journal as well as be made available on ClinicalTrials.gov.

\section{Author affiliations}

${ }^{1}$ Department of Internal Medicine - Cardiology Section, Holbaek Hospital, Holbaek, Region Zealand, Denmark

${ }^{2}$ Department of Regional Health Research, University of Southern Denmark Faculty of Health Sciences, Odense, Denmark

${ }^{3}$ Department of Cardiology, Odense University Hospital, Odense C, Denmark ${ }^{4}$ Copenhagen Trial Unit, Centre for Clinical Intervention Research, Rigshospitalet, Copenhagen University Hospital, Capital Region of Denmark, Copenhagen, Denmark ${ }^{5}$ Department of Cardiology, Hvidovre University Hospital, Hvidovre, Denmark ${ }^{6}$ Department of cardiology, Zealand University Hospital Roskilde, Roskilde, Region Zealand, Denmark
Acknowledgements The authors would like to thank the patient advisory committee at Holbaek Hospital. We would also like to thank Lise Pedersen and Bo Christensen from the Department of Clinical Biochemistry as well as Palle Lyngsie Pedersen from the Region of Zealand biobank for their help in planning the logistics surrounding the biobank.

Contributors JF, JCJ, AB, UD, UJOG, WB, MHO, ODP and IR participated integrally in the study design. CG provided vital advice on trial conduct. EEN and FS-H designed the echocardiography plan. $\mathrm{MHO}$ designed the plan for analysis of biomarkers. JF, JCJ and AB drafted the initial manuscript. All other authors provided critical revision and approved the final manuscript.

Funding The trial was initiated by clinicians at the participating hospitals. The research salary for research nurses is partly funded by the Region of Southern Denmark and Region Zealand joint research fund 2018 for year 1 . The salary of the lead author for years 2 and 3 are provided by the Danish Heart foundation grant number 19-R134-A8959-22123. The salary for year 1 is granted by the University of Southern Denmark. The participating departments support the trial by dedicating work hours of the other investigators, supportive staff, logistical support and administrative support. The trial is investigator initiated. Holbaek Hospital is the sponsor, and the Region Zealand is the data controller. The study sponsors and funders had no influence on design; collection, management, analysis and interpretation of data; writing of the report; and the decision to submit the report for publication. The Danish Heart Foundation requires to be notified by email when a publication is accepted. Roles and responsibilities of additional parties are described in supplementary file 8 .

Competing interests JBF (PI), IR, WB, EEN, FS-H, ODP, UG, CG and JCJ report no competing interests. $\mathrm{MHO}$ reports grants from Novo Nordic Foundation outside the submitted work. AB reports personal fees from Bayer, grants from Biotronik, personal fees from Boehringer Ingelheim, personal fees from Bristol-Myers Squibb, personal fees from MSD, grants from Theravance, outside the submitted work. UD reports a research grant from Bayer, personal fees from Pfizer, member of advisory board for Boehringer Ingelheim, member of advisory board for Merck, outside the submitted work.

Patient consent for publication Not required.

Provenance and peer review Not commissioned; externally peer reviewed.

Supplemental material This content has been supplied by the author(s). It has not been vetted by BMJ Publishing Group Limited (BMJ) and may not have been peer-reviewed. Any opinions or recommendations discussed are solely those of the author(s) and are not endorsed by BMJ. BMJ disclaims all liability and responsibility arising from any reliance placed on the content. Where the content includes any translated material, BMJ does not warrant the accuracy and reliability of the translations (including but not limited to local regulations, clinical guidelines, terminology, drug names and drug dosages), and is not responsible for any error and/or omissions arising from translation and adaptation or otherwise.

Open access This is an open access article distributed in accordance with the Creative Commons Attribution Non Commercial (CC BY-NC 4.0) license, which permits others to distribute, remix, adapt, build upon this work non-commercially, and license their derivative works on different terms, provided the original work is properly cited, appropriate credit is given, any changes made indicated, and the use is non-commercial. See: http://creativecommons.org/licenses/by-nc/4.0/.

ORCID iD

Joshua Buron Feinberg http://orcid.org/0000-0003-1975-9300

\section{REFERENCES}

1 Pistoia F, Sacco S, Tiseo C, et al. The epidemiology of atrial fibrillation and stroke. Cardiol Clin 2016;34:255-68.

2 Camm AJ, Lip GY, De Caterina R. 2012 focused update of the ESC guidelines for the management of atrial fibrillation: an update of the 2010 ESC guidelines for the management of atrial fibrillation. Europace 2012;14:1385-413.

3 Stewart S, Hart CL, Hole DJ, et al. A population-based study of the long-term risks associated with atrial fibrillation: 20-year follow-up of the Renfrew/Paisley study. Am J Med 2002;113:359-64.

4 Benjamin EJ, Wolf PA, D'Agostino RB, et al. Impact of atrial fibrillation on the risk of death: the Framingham heart study. Circulation 1998;98:946-52.

5 Rahman F, Wang N, Yin X, et al. Atrial flutter: clinical risk factors and adverse outcomes in the Framingham heart study. Heart Rhythm 2016;13:233-40. 
6 Healey JS, Oldgren J, Ezekowitz M, et al. Occurrence of death and stroke in patients in 47 countries 1 year after presenting with atrial fibrillation: a cohort study. Lancet 2016;388:1161-9.

7 Wolf PA, Abbott RD, Kannel WB. Atrial fibrillation as an independent risk factor for stroke: the Framingham study. Stroke 1991;22:983-8.

8 Odutayo A, Wong CX, Hsiao AJ, et al. Atrial fibrillation and risks of cardiovascular disease, renal disease, and death: systematic review and meta-analysis. BMJ 2016;354:i4482.

9 AS G, Hylek EM, Phillips KA. Prevalence of diagnosed atrial fibrillation in adults: national implications for rhythm management and stroke prevention: the anticoagulation and risk factors in atrial fibrillation (atria) study. JAMA 2001;285:2370-5.

10 Stewart S, Murphy N, Walker A, et al. Cost of an emerging epidemic: an economic analysis of atrial fibrillation in the UK. Heart 2004;90:286-92

11 Mozaffarian D, Benjamin EJ, AS G. Heart disease and stroke statistics-2016 update: a report from the American heart association. Circulation 2016;133:e38-60.

12 January CT, Wann LS, Alpert JS. 2014 AHAVACC/HRS guideline for the management of patients with atrial fibrillation: a report of the American College of Cardiology/American heart association Task force on practice guidelines and the heart rhythm Society. J Am Coll Cardiol y 2014;64:e1-76.

13 Sethi NJ, Feinberg J, Nielsen EE, et al. The effects of rhythm control strategies versus rate control strategies for atrial fibrillation and atrial flutter: a systematic review with meta-analysis and trial sequential analysis. PLoS One 2017;12:e0186856.

14 Kirchhof P, Benussi S, Kotecha D, et al. 2016 ESC guidelines for the management of atrial fibrillation developed in collaboration with EACTS. Eur Heart J 2016;37:2893-962.

15 Andrade JG, Aguilar M, Atzema C, et al. The 2020 Canadian cardiovascular Society/Canadian heart rhythm Society comprehensive guidelines for the management of atrial fibrillation. Canadian Journal of Cardiology 2020;36:1847-948.

16 Van Gelder IC, Groenveld HF, Crijns HJGM, et al. Lenient versus strict rate control in patients with atrial fibrillation. $N$ Engl J Med 2010;362:1363-73.

17 Groenveld HF, Crijns HJ, Van den Berg MP. The effect of rate control on quality of life in patients with permanent atrial fibrillation: data from the race II (rate control efficacy in permanent atrial fibrillation II) study. J Am Coll Cardiol 2011;58:1795-803.

18 Van Gelder IC, Crijns HJGM, Blanksma PK, et al. Time course of hemodynamic changes and improvement of exercise tolerance after cardioversion of chronic atrial fibrillation unassociated with cardiac valve disease. Am J Cardiol 1993;72:560-6.

19 Nerheim P, Birger-Botkin S, Piracha L, et al. Heart failure and sudden death in patients with tachycardia-induced cardiomyopathy and recurrent tachycardia. Circulation 2004;110:247-52.

20 Anter E, Jessup M, Callans DJ. Atrial fibrillation and heart failure: treatment considerations for a dual epidemic. Circulation 2009;119:2516-25.

21 Mulder BA, Van Veldhuisen DJ, Crijns HJGM, et al. Lenient vs. strict rate control in patients with atrial fibrillation and heart failure: a posthoc analysis of the race II study. Eur J Heart Fail 2013;15:1311-8.

22 Jakobsen JC, Gluud C, Wetterslev J, et al. When and how should multiple imputation be used for handling missing data in randomised clinical trials - a practical guide with flowcharts. BMC Med Res Methodol 2017;17:162.

23 Reynolds MR, Ellis E, Zimetbaum P. Quality of life in atrial fibrillation: measurement tools and impact of interventions. J Cardiovasc Electrophysiol 2008;19:762-8.

24 Post M. Definitions of quality of life: what has happened and how to move on. Top Spinal Cord Inj Rehabil 2014;20:167-80.
25 Kotecha D, Ahmed A, Calvert M, et al. Patient-Reported outcomes for quality of life assessment in atrial fibrillation: a systematic review of measurement properties. PLoS One 2016;11:e0165790.

26 Kotecha D, Breithardt G, Camm AJ, et al. Integrating new approaches to atrial fibrillation management: the 6th AFNET/EHRA consensus conference. Europace 2018;20:395-407.

27 Spertus J, Dorian P, Bubien R, et al. Development and validation of the atrial fibrillation effect on quality-of-life (AFEQT) questionnaire in patients with atrial fibrillation. Circ Arrhythm Electrophysiol 2011;4:15-25.

28 Maglio C, Sra J, Paquette M. Measuring quality of life and symptom severity in patients with atrial fibrillation. Pacing Clin Electrophysiol 1998;21:839.

29 Crosby RD, Kolotkin RL, Williams GR. Defining clinically meaningful change in health-related quality of life. J Clin Epidemiol 2003;56:395-407.

30 Dorian P, Burk C, Mullin CM, et al. Interpreting changes in quality of life in atrial fibrillation: how much change is meaningful? Am Heart $J$ 2013;166:381-7.

31 Jakobsen JC, Gluud C, Winkel P, et al. The thresholds for statistical and clinical significance - a five-step procedure for evaluation of intervention effects in randomised clinical trials. BMC Med Res Methodol 2014;14:34.

32 World Medical Association Declaration of Helsinki. Ethical principles for medical research involving human subjects. JAMA 2013;310:2191-4.

$33 \mathrm{ICH}$ Harmonised Guideline. Integrated addendum to ICH E6(R1). Guideline for good clinical practice E6(R2), 2016. Available: https:// database.ich.org/sites/default/files/E6_R2_Addendum.pdf

34 Kirchhof P, Camm AJ, Goette A, et al. Early rhythm-control therapy in patients with atrial fibrillation. N Engl J Med 2020;383:1305-16.

35 Gillis AM. A sober reality? alcohol, abstinence, and atrial fibrillation. N Engl J Med 2020;382:83-4.

36 Ware JE, Sherbourne CD. The mos 36-item short-form health survey (SF-36). I. conceptual framework and item selection. Med Care 1992;30:473-83.

37 Alam M, Wardell J, Andersson E, et al. Characteristics of mitral and tricuspid annular velocities determined by pulsed wave Doppler tissue imaging in healthy subjects. J Am Soc Echocardiogr 1999;12:618-28.

38 Mitchell C, Rahko PS, Blauwet LA, et al. Guidelines for performing a comprehensive transthoracic echocardiographic examination in adults: recommendations from the American Society of echocardiography. J Am Soc Echocardiogr 2019;32:1-64.

39 Wokhlu A, Monahan KH, Hodge DO. Long-term quality of life after ablation of atrial fibrillation the impact of recurrence, symptom relief, and placebo effect. J Am Coll Cardiol 2010;55:2308-16.

40 Dorian P, Paquette M, Newman D, et al. Quality of life improves with treatment in the Canadian trial of atrial fibrillation. Am Heart $J$ 2002;143:984-90.

41 Kahan BC, Morris TP. Improper analysis of trials randomised using stratified blocks or minimisation. Stat Med 2012;31:328-40.

42 Jakobsen JC, Tamborrino M, Winkel P. Count data analysis in randomised clinical trials. J Biomet Biostat 2015;6:227.

43 International Committee of Medical Journal Editor. Recommendations. defining the role of authors and contributors, 2020. Available: http://www.icmje.org/recommendations/browse/ roles-and-responsibilities/defining-the-role-of-authors-andcontributors.htm

44 Higgins JPT, Thomas J, Chandler J. Cochrane Handbook for systematic reviews of interventions version 6.1 (updated September 2020. London: Cochrane, 2020. www.training.cochrane.org/ handbook 